\title{
The uncertain but critical role of demand reduction in meeting long-term energy decarbonisation targets
}

\author{
Steve Pye, Will Usher and Neil Strachan
}

UCL Energy Institute, University College London

14 Upper Woburn Place, London, WC1H 0NN, United Kingdom

Tel: +44 (0)2031085989

e-mail: s.pye@ucl.ac.uk

\section{Abstract}

Endogenous demand responses for energy services, resulting from changing prices, have long been characterised in energy systems models. However, the uncertainty associated with such demand responses, modelled through the use of price elasticities, has often been ignored. This is problematic for two key reasons - elasticity factors used in models are highly uncertain due to the limited evidence base, while at the same time, demand response has been observed as a critical mechanism for meeting longer term climate mitigation targets. This paper makes two important contributions to improving understanding of the role of price-induced demand response. Firstly, it attempts to address the problem of unsatisfactory elasticity input assumptions by undertaking an up-to-date review of the literature. Secondly, the role of demand response under uncertainty is assessed using a probabilistic approach, focusing on its contribution to mitigation. The paper highlights that demand response does play a critical role in mitigation, ensuring a more cost-effective transition to a low carbon energy system. Crucially, the uncertainties associated with price elasticities do not weaken this finding. The transport sector is the driver of this demand response leading to important implications for policy and the focus of demand side interventions.

Keywords: Energy demand response; climate change mitigation; energy system modelling JEL codes: Q4

An earlier version of this paper was submitted to and accepted at the 33rd edition of the International Energy Workshop (IEW), Beijing, 4-6 June 2014. 


\section{Introduction}

The challenge of meeting medium to long term decarbonisation targets cost-effectively not only requires the large scale uptake of low carbon technologies and fuels but also behaviour change. The role of behaviour change, alongside technological solutions, has been an emerging theme of many international and national modelling assessments. In a review of low carbon transition modelling analyses, Strachan et al. (2008) concludes that low carbon scenarios are technologically feasible given expected progress in low-carbon measures and the behavioural change required to adopt technologies and complement them with emissions reductions. This conclusion emerges strongly from other high profile international modelling studies such as the Energy Technology Perspective (ETP, IEA 2012) and the Global Energy Assessment (GEA, Nakićenović (Ed.) 2012). The ETP highlights the important role of modal shift in the transport sector and preferences for vehicle type, and the impacts of behaviour on energy use in residential buildings. The GEA highlights the need for a change in culture and lifestyles as part of the wider strategy for a more sustainable, low carbon energy system.

Energy systems models (ESMs) have emerged as an important energy modelling approach for exploring pathways for meeting long term decarbonisation targets. Nowhere has their systematic use for national strategy development been as well demonstrated as in the UK. Since 2000, following the landmark publication by the RCEP (2000), proposing a $60 \%$ national decarbonisation goal, energy system models have provided supporting analysis to each energy strategy (Strachan et al. 2009), strategies which have led to the establishment of a legislative act mandating a carbon reduction target of $80 \%$ reduction in GHG emissions in 2050, relative to 1990 levels, and a set of ambitious mid-term carbon budgets (DECC 2011). All modelling analyses have highlighted the affordability of ambitious low carbon targets but the requirement for investment in a diverse mix of technology options and the requirement for behavioural change (Ekins et al. 2013).

One feature often used to represent a key aspect of behaviour change in ESMs is price elastic demands. This allows for demand for energy services to be endogenously determined based on changing prices for those services. In the case of climate targets, investment in low carbon technologies and fuels drive up prices for energy services, leading to reductions in demand. The role of demand reduction under 
carbon targets has been identified as critical for delivering ambitious mitigation targets, particularly in sectors where technical supply-side measures are higher cost or their potential is limited. Anandarajah et al. (2009) estimate demand reductions of up to $25 \%$ for residential and industrial sectors under the UK's $80 \%$ reduction target in 2050 . A recent UK modelling study suggests that the technical feasibility of meeting the $80 \%$ decarbonisation goal would not be possible without demand reduction (AEA 2011). Chen et al. (2007) underline the importance of demand reduction in their analysis of mitigation costs in China, estimating a resulting $60 \%$ reduction in marginal abatement costs in 2020 . Other analyses also support the importance of demand reductions in climate mitigation both in the UK (AEA 2008), and globally (Kesicki and Anandarajah 2011). Given the criticality of demand reductions to a low carbon system transition, assumptions need to be as robust as possible.

However, two key problems are associated with the modelling of demand response in ESMs. Firstly, elasticity assumptions have often been poorly defined, unclear what they represent (short or long run, final energy demand or energy service demand), been subject to limited review, and taken from a limited empirical evidence base. Secondly, as a result, these model input assumptions are highly uncertain. In this paper, the characterisation of price-driven demand response is improved via an up-to-date review of the literature, and innovative probabilistic implementation of price elasticity uncertainties in the UK Energy Systems Modelling Environment (ESME) model. The improved characterisation of demand response in ESME allows for more robust insights into its role across different sectors and the tradeoffs with supply-side options (including technical energy efficiency measures), and the impact of uncertainty associated with demand side response in a systems context.

Section 2 provides a review of the literature, briefly examining the role of demand response as highlighted in recent mitigation modelling analyses, and then focuses on a review of the literature on price elasticity assumptions. It then describes the energy system modelling framework used for exploring demand response, the ESME model, and the approach to modelling price elasticities. Section 3 present the results of the analysis, followed by a discussion of the insights from the analysis in section 4. Finally, section 5 discusses the implications of the research for policy and outlines further research needs. 


\section{Material and methods}

\subsection{Literature Review: Price elasticities for energy service demands}

\subsubsection{The challenges of implementing demand response in ESMs}

The strength of energy systems models (ESMs) is their integrated approach to modelling of energy supply and demand, providing rich detail on the technologies and fuels that will be required to meet future energy demands under a range of possible scenarios. Well known models, such as MARKAL/TIMES (Fishbone et al. 1981, Seebregts et al. 2002) and MESSAGE (Messner and Schrattenholzer 2000), use linear programming to assess cost-optimal mixes of technologies, based on technical costs associated with energy system development. The focus of such models on supply-side representation draws attention to the deficiencies in the representation of demand side and behavioural factors, as highlighted by Hourcade et al. (2006), and Schafer (2012).

However, the inclusion of price elastic demands, a key feature of representing price-driven behaviour, has long been a feature of ESMs. First implemented in MARKAL models by Loulou and Lavigne (1996), it is now a feature of most ESMs (Bhattacharyya and Timilsina 2009), and is described in more detail in section 3. It allows for the modelling of endogenous trade-offs between investment in low carbon technologies and fuels, including end use sector energy efficiency and conservation, and the loss of welfare associated with reducing demand (due to price increases, not through voluntary reductions). Most ESMs use own price elasticities, which measure the percentage change in the quantity demanded of a given energy service as a result of a percentage change in its price. Cross price elasticities, on the other hand, measure the change in quantity demanded of a given energy service $\mathrm{X}$ as a result of a change in price of energy service Y. The most common application of cross-price elasticities relates to transport demand; however, few ESMs explicitly use cross-price elasticities (Schafer 2012). ${ }^{1}$

Determining own price elasticities, the focus of this paper, and their associated uncertainty for use in ESMs is challenging, as noted by Anandarajah et al. (2009) and by Sorrell et al. (2009). A key difficulty

${ }^{1}$ The implementation of cross-price elasticities is described in the MICRO version of MARKAL/TIMES models (Seebregts et al. 2002) although no papers in the literature describe this model implementation for analysis. 
in arises due to the type of elasticity factor required. Sorrell et al. (2009) usefully differentiates between the following elasticity types (where $D$ is demand, $E S$ is energy services, $P$ is price) -1 ) elasticity of $D$ for $E S$ with respect to $P$ of $E S, 2$ ) elasticity of $D$ for $E S$ with respect to $P$ of energy and 3) elasticity of $D$ for energy with respect to $P$ of energy. For ESMs, values for 1) tend to be required as demands are usually specified as energy services while much of the literature focuses on 2) and 3). In addition, studies often analyse short run elasticities while for ESMs undertaking longer term analysis, long run elasticities are more appropriate, to represent the effect of price changes over a longer period (5-10 years), allowing for more radical shift in choices e.g. change in technology stock.

Secondly, the range of estimates for different energy demands is large, with many of the studies using different estimation approaches (Sorrell and Dimitropoulos 2007). Differences also result from geographic location of studies, and the types of demands being assessed. For example, transport sector elasticities can vary depending on trip purpose, income group, spatial context (urban versus rural), and choice of alternatives (Litman 2013). Thirdly, elasticity estimates are based on historical observation using econometric approaches, capturing market imperfections and policy initiatives. Optimisation models however are forward looking, assume perfect market conditions and are often run with policies omitted (Boonekamp 2007). Finally, and related to the above point, models only provide a partial representation of the 'real world'. The observed relationship between price and demand may be based on a range of choices that may not be present in the model. For a given elasticity, the demand response in a poorly specified model sector may therefore be significantly larger than observed empirically.

Price elasticities in UK ESM analyses (most using the MARKAL model framework) have to date been based on a limited number of model-based sources (Kannan et al. 2007), reflecting the challenge in sourcing these type of data. Whilst these sources are presumably based on a review of the evidence, it is not possible to determine how they have been compiled. They are from different model types, and conceivably represent different types of price elasticities (as per the typology above). To date, based on the knowledge of the authors, these assumptions have never been critiqued or reviewed. The problem of attributing the source of these data has steered this research to undertaking a review of price elasticity assumptions, which is summarised by sector below. 


\subsubsection{Road transport price elasticities}

In the field of price elasticity estimation, the transport sector is the most widely studied. In recent years, the most comprehensive UK-based reviews for road transport include Goodwin et al. (2004) and Graham and Glaister (2004). Goodwin et al. (2004) reviewed 69 different studies, most of which focused on demand for fuel in car vehicles; however, a high proportion (20\%) also included estimates of change in demand for travel given an increase in energy prices. The review focused on UK and comparable countries, with a large proportion (40-50\%) of the estimates from US studies ( $\mathrm{n}=63$ ) and a similar number from European countries (18\% of estimates were from the UK). An interesting observation was that price elasticities were lower in the US studies, compared to European-based analyses.

Long run effects were always observed to be higher than short run effects (by a factor of 2-3), due to the greater ability (over a longer period) to shift to more efficient vehicles or different modes. The higher reduction in fuel consumption compared to vehicle-km (in Table 1) implies a shift towards the purchase of more energy efficient vehicles. The vehicle stock value, whilst more uncertain based on the limited number of studies reviewed, suggests that car ownership may also be affected by price increases. From a review of the international literature, estimates by Graham and Glaister (2004) are broadly consistent. Their review of elasticities as it relates to car travel demand draws heavily from a wide ranging review of the European literature by De Jong and Gunn (2001), while those relating to fuel consumption are based on a much larger international evidence base of 113 studies.

Table 1. Estimates of price elasticities of demand for private road vehicles (Goodwin et al. 2004, Graham and Glaister 2004)

\begin{tabular}{|l|l|l|l|}
\hline Dependent variable & Short term & \multicolumn{2}{|l|}{ Long term } \\
\hline Goodwin et al (2004) & Mean & Mean & Range \\
\hline Fuel consumption & -0.25 & -0.64 & $0,-1.81$ \\
Vehicle-km & -0.1 & -0.29 & $-0.63,-0.1$ \\
Vehicle stock & -0.08 & -0.25 & $-0.63,-0.1$ \\
\hline Graham and Glaister (2004) & & & \\
\hline Fuel consumption & -0.25 & -0.77 & \\
Car-km & -0.16 & -0.26 & \\
Car trips & -0.16 & -0.19 & \\
\hline
\end{tabular}


We have proposed a central estimate of -0.3 for use in the model, with a range of -0.15 to -0.5 (similar to that suggested by Goodwin et al. 2004). We recognise that the values in the review are using fuel prices, and that to use these values as a proxy for the cost of meeting car demand ignores that investment costs account for a larger share of costs in the model. Studies that include a generalised costs metric tend to have higher long run elasticities ( - 0.7$)$ but include a range of costs not included in ESME such as travel time costs, road pricing etc. (Litman 2013).

For road freight, the conclusion in Goodwin et al. (2004), based on a limited evidence base, is that commercial vehicle operators are likely to be less sensitive to fuel price changes. This is because direct fuel costs are likely to be a smaller proportion of total freight costs, which are part of a broader manufacturing and distribution process. Goods have to be transported, and there is limited substitutability to other modes. The review by Graham and Glaister (2004) suggests that the observations outlined by Goodwin et al. (2004) are by no means definitive, that in fact road freight is not necessarily price inelastic although the evidence base, largely from North American studies, is very weak. They highlight the large variation, noting the likely causes being due to different estimation approaches, data used, type of freight, and definition of demand; however, understanding this variation is difficult due to the small number of studies.

For ESME, it is assumed that that freight elasticities will be lower than for road passenger travel - a central estimate of -0.2 is assumed across $\mathrm{MGV}$ and $\mathrm{HGV}$ categories, with a range of -0.05 to -0.3 , while LGVs are slightly more elastic, with a range of -0.1 to -0.35 , with a central estimate of -0.25 . These estimates are in line with those used in the Department for Transport's National Transport Model (NTM) (DfT 2010).

While much of the elasticity estimates for private road transport are derived from fuel price studies, public transport elasticities are typically based on fares. In a wide ranging review by Paulley et al. (2006), bus fare elasticity averages were estimated at around $-0.4,-0.56$ and -1.0 in the short, medium and long run respectively. For long run elasticities, the range was -0.85 to -1.32 (based on a much smaller sample of three studies). Earlier studies by Dargay and Hanly (2002) estimated lower long run 
elasticities of around -0.62. In a review of transit elasticities, Litman (2013) cites estimates from UK studies that suggest fare elasticities of around -0.7. In one of these studies (by Dargay and Hanly, $1999^{2}$ and not reviewed directly for this paper) the importance of urban versus non-urban is once again highlighted, with the urban estimate range of -0.4 to -0.6 , and non-urban range of -0.8 to -1.0 . This reflects less reliance on buses, poorer service levels and greater feasibility of using cars as an alternative in non-urban areas. In ESME, a central elasticity assumption is proposed of -0.7 , with a range of -0.5 to -1.0 .

\subsubsection{Non-road transport sector price elasticities}

Dargay (2010) estimates a long run price elasticity of -1.0 for passenger rail, stating that this value is well-supported by a large body of evidence. In the same analysis, elasticity values are disaggregated by journey purpose and distance. As for other modes, business and commuting demands are inelastic (around -0.5) while leisure / holiday travel is more elastic (<-1.0). Paulley et al. (2006) highlight the temporal dimension to rail travel, noting the more elastic demand at off-peak times than peak times (short run values of -0.65 and -0.42 respectively). By comparison, DfT use a central estimate of -0.7 in their modelling. For ESME, a central estimate of -0.8 is proposed, and a range of -0.6 to -1.1 . To date, no studies have been found relating to rail freight. In the absence of evidence, we have assumed this demand to be highly inelastic; ${ }^{3}$ however, it could be that demand response for rail freight is relatively elastic, with many goods able to be transported by road.

Elasticities for aviation demand are primarily based on studies that capture the change in demand in response to changes in fares. Estimates tend to vary significantly, again due to differences in methods, and because a large number of factors come into play creating a complex picture (Dargay et al. 2006, Litman 2013), including type of travel (business versus leisure), mode substitution and destination substitution. In a review by Dargay et al. (2006), demand was shown to be far more responsive to fare changes over time (as per other modes), with significantly higher price elasticities in the long run (2-3 times higher than short run elasticities). Estimates in the range of -0.5 to -1.5 are typical from the studies

\footnotetext{
2 Joyce Dargay and Mark Hanly (1999), Bus Fare Elasticities. ESRC Transport Studies Unit, University College London 3 The same approach has been taken for the marine sector.
} 
reviewed, with average values at unity. In forecasting future aviation demand, DfT (2013) cite a range of fare price elasticities depending on the type of market and propose an overall elasticity of -0.6 for international travel, with strong differentiation between business and leisure travel in particular $(-0.2$ for business, -0.7 for leisure). Domestic demand is assumed to be less elastic, with a value of -0.5 , which is surprising given the assumed greater scope for substitution between modes (as suggested in other studies $^{4}$ ). For ESME, a central estimate of -0.6 has been assumed for international travel and -0.7 for domestic travel. The proposed ranges are based on DfT (2013) estimates and typical ranges cited in the Dargay et al. (2006) review, and by InterVISTAS (2007).

\subsubsection{Buildings sector price elasticities}

While the evidence base is much more limited for the buildings sector, there are a number of studies that provide a basis for estimates for use in a model. In a review of econometric studies by Sorrell and Dimitropoulos (2007), differences between long and short run elasticities were not observed across household sector elasticities, as observed in the transport sector. However, similar to transport sector demands, price elasticities tend to be inelastic (>-1.0). In addition, elasticities tend to vary with price level, with higher prices encouraging higher elasticities, and be asymmetric, with the response to increasing prices being greater than to falling prices.

Guertin et al. (2003) estimated long run price elasticities for three categories of household energy services - space heating, hot water, and appliances and lighting, and across different income groups in Canada. The average elasticity for the combined services is estimated at -0.35 , with low income household demand estimated to be $70 \%$ more elastic. Klein (1988) used data from 2000 US households to develop a model analysing household production and consumption of space heat, using a system of simultaneous equations to estimate the total cost of space heat, the share of energy costs in space heat production, and demand for the energy service. The analysis estimates short run elasticities for space heating demand (based on the costs of providing the energy service) of -0.25 , and a long run elasticity of -0.47 , stating that such estimates are in the range of other studies published at the time. The extensive

\footnotetext{
${ }^{4}$ Based on Gillen, D.W, Morrison, W.G, Stewart, C (2003) Air travel demand elasticities: Concepts, Issues and Measurement, Department of Finance, Government of Canada (cited in Dargay et al. 2006)
} 
UKERC-funded study on the rebound effect (detailed in Sorrell 2007, Sorrell and Dimitropoulos 2007, and summarised in Sorrell et al. 2009) provides a useful review of econometric studies on price elasticities for household energy consumption. Estimates for the rebound effect from OECD studies (including Klein 1988 and Guertin et al. 2003) from the review are reproduced in Table 2. The degree of confidence in estimates is stated as significantly lower than for the transport sector (where it is considered 'high').

Table 2. Estimates of the Rebound effect for different household energy services (Sorrell 2007, Sorrell et al. 2009)

\begin{tabular}{|l|l|l|l|l|}
\hline Energy service & $\begin{array}{l}\text { Range from } \\
\text { evidence base }\end{array}$ & 'Best guess' & No. of studies & $\begin{array}{l}\text { Degree of } \\
\text { confidence }\end{array}$ \\
\hline Space heating & $1.4-60 \% \%$ & $10-30 \%$ & 9 & Medium \\
\hline Space cooling & $1-26 \%$ & $1-26 \%$ & 2 & Low \\
\hline Other services & $0-49 \%$ & $<20 \%$ & 3 & Low \\
\hline
\end{tabular}

The evidence base points to the use of price elasticities (central estimates) of between -0.2 and -0.3 for space heating. In ESME, space heating is bundled with water heating and lighting (in the 'dwelling' demands). For other household energy services, a central estimate of -0.15 is assumed, reflecting fewer opportunities (and / or greater reluctance) to reduce cooking energy services and appliance use. For cooling services, this estimate is in line with the UKERC review (Sorrell 2007).

The evidence base for commercial buildings is very limited. Different US studies (Paul et al. 2009, Bernstein and Griffin 2006) provide information on differences between commercial sector elasticities for electricity consumption; however, such elasticities reflect technical solutions to reducing energy consumption as opposed to reducing energy service demands (ESD), and are not appropriate to use in a model such as ESME. Firms in commercial building or public sector buildings may be able to reduce specific energy service demands, such as lighting and appliance use, through behaviour change programmes. However, for other energy services such as space heating and cooling, this is more difficult, with workplaces wanting to maintain specified levels of thermal comfort. Due to the lack of available data on ESD responsiveness to prices, a conservative central estimate of -0.1 has been assumed (with a range of $0--0.15$ ). 


\subsubsection{Industry sector}

Elasticities for use in the model's industry sector should represent the change in industrial output as a function of the cost of providing energy services for production (as model demands are a proxy of sector output). The implication of such a demand response is that the cost of energy services impact on the economics of production. In terms of carbon mitigation, this response differs from other sectors, in that it is not incentivising behaviour change but rather reducing production, or shifting production abroad. Determining elasticity factors to represent this relationship is contentious because energy services may account for a small proportion (or not) of overall production costs. In determining the elasticity factor, the value for each subsector needs to take account of the share of energy costs in the total costs of production, the competitiveness impacts on production (based on exposure to international competition), and the ability to relocate to other regions (where costs may be cheaper). Under carbon targets, the reduction in production could be attributed as carbon leakage, either through loss of competitiveness (production leakage) or through relocation (investment leakage).

The difficulties associated with using price elasticities for industrial energy services are fourfold. Firstly, there is a need to understand fuel costs as a share of the overall costs of production, with those sector incurring higher costs more elastic to price increases. ${ }^{5}$ Secondly, increasing costs for industries that are more exposed to intense competition could have a larger impact in terms of production. Thirdly, there is an issue with characterisation of the industry sector in ESME. Data on supply side options to allow industry to reduce fuel costs are limited, and therefore the role of demand side response (irrespective of elasticity assumptions) may be overplayed. Finally, the data to characterise price elasticities is very limited. Where price elasticities are estimated for different industry sectors, these are in regard to industry response (through investment) to reduce fuel costs via investment in energy efficiency measures or fuel switching, features captured on via supply side modelling.

Recent UK analyses using MARKAL (for example, Anandarajah et al. 2009) have used relatively high long run elasticity factors for industry. However, it is not clear what type of demand-price relationship

\footnotetext{
${ }^{5}$ The Energy Intensive Users Group (EIUG) estimates that energy related production costs as a percentage of total costs currently amount to around $20-25 \%$ for the steel, paper, glass and cement industries, $40 \%$ for aluminium smelting and as high as 70\% for industrial gases. (Civitas 2010, http://www.civitas.org.uk/pdf/EnergyPolicyApril2010.pdf)
} 
such factors represent i.e. price response for energy demand or energy service demand. Given the poor evidence base, and the difficultly of introducing elasticity factors into an ESM for the reasons given above, price elasticities in ESME are assumed to be highly inelastic, using a value of - 0.05 , and thereby severely restricting opportunities for endogenous demand response.

\subsubsection{Summary}

The review of elasticity assumptions shows the significant variability in the nature of the evidence across different demands. While providing an improved basis for characterising demand response in ESME, it also highlights the value in using a probabilistic approach to capture this uncertainty. Another important feature of the studies reviewed is that elasticity values differ considerably when disaggregated demand categories are considered e.g. trip purpose / destination in aviation. This points towards the use of more highly disaggregated demands in ESMs in future analysis. The values summarised in Table 3 below represent averaged demand responses, as elasticities are applied to aggregated energy service demands in ESME. Other assumptions related to the use of these values in ESME are described in the following methodology section.

Table 3. Elasticity input parameters by energy service demand

\begin{tabular}{|l|l|l|l|l|}
\hline ESD Name & Sector & Low & Central & High \\
\hline Aviation Domestic Passenger & Transport & -0.50 & -0.70 & -1.50 \\
\hline Aviation International Passenger & Transport & -0.40 & -0.60 & -1.00 \\
\hline Rail Passenger (electric and diesel) & Transport & -0.60 & -0.80 & -1.10 \\
\hline Rail Freight & Transport & -0.01 & -0.03 & -0.05 \\
\hline Road Passenger Car (2 size classes) & Transport & -0.15 & -0.30 & -0.50 \\
\hline Road Passenger Bus & Transport & -0.50 & -0.70 & -1.00 \\
\hline $\begin{array}{l}\text { Road Freight Goods Vehicle (heavy and } \\
\text { medium)) }\end{array}$ & Transport & -0.05 & -0.20 & -0.30 \\
\hline Road Freight Light Goods Vehicle & Transport & -0.10 & -0.25 & -0.35 \\
\hline Maritime International Freight & Transport & -0.01 & -0.03 & -0.05 \\
\hline Maritime Domestic Freight & Transport & -0.01 & -0.03 & -0.05 \\
\hline $\begin{array}{l}\text { Dwellings (3 density types - high, medium, } \\
\text { low) }\end{array}$ & Residential & -0.10 & -0.25 & -0.40 \\
\hline Appliances & Residential & -0.05 & -0.15 & -0.30 \\
\hline Cooking & Residential & -0.05 & -0.15 & -0.30 \\
\hline Air Conditioning & Residential & -0.05 & -0.15 & -0.30 \\
\hline Commercial Floorspace & Comm. / Public sector & -0.01 & -0.10 & -0.15 \\
\hline Public Floorspace & Comm. / Public sector & -0.01 & -0.10 & -0.15 \\
\hline Industry (8 subsectors) & Industry & -0.01 & -0.03 & -0.05 \\
\hline
\end{tabular}




\subsection{Methodology: Modelling demand response in an ESM}

\subsubsection{The ESME model}

ESME (Energy Systems Modelling Environment), developed by the Energy Technologies Institute (ETI), is a fully integrated ESM, used to determine the role of different low carbon technologies required to achieve the UK's mitigation targets. ${ }^{6}$ The model has been used in this capacity by the UK Department for Energy and Climate Change (DECC) and the UK Committee on Climate Change (CCC) (CCC 2011, CCC 2013, DECC 2011). Built in the AIMMS environment, it uses linear programming to assess cost-optimal technology portfolios. The uncertainty around cost and performance of different technologies and resource prices is captured via a probabilistic approach, using Monte Carlo sampling techniques. The focus of uncertainty is on technology investment costs in the power and transport sectors, fuel costs and resource potential e.g. biomass imports. ${ }^{7}$

The representation of energy demand sectors is typical of other ESMs, and shown in Table 3, alongside corresponding demands. The model endogenously determines how to meet these demands in the most cost-optimal manner, through investment end use technologies (including efficiency measures), production and supply of different energy forms, and via reductions in the demand for energy services (as described in the next section). In the household sector, a rich characterisation of low carbon technologies is provided, particularly for heat pumps, district heating (incl. infrastructure) and building fabric retrofit. The transport sector also incorporates the key low carbon technologies, and the different infrastructure required to deliver alternative fuels e.g. electricity charging infrastructure and hydrogen networks. The industry sector is characterised more simply, focusing on efficiency gains, fuel switching measures and carbon capture and storage (CCS). Transformation sectors (power generation, hydrogen production, biofuel production) represent the key low carbon technologies, and associated infrastructures (to enable inter-node transmission). Primary resource supply is characterised by commodity price and resource availability, with no distinction between imports and domestic indigenous production (except for biomass).

\footnotetext{
${ }^{6} \mathrm{~A}$ full description of the model can be found at http://eti.co.uk/downloads/related documents/ESME Modelling Paper.pdf

7 ESME model version 3.1 with elastic demand extension has been used for this analysis.
} 


\subsubsection{Elastic demand formulation in ESME}

In the elastic demand formulation of ESME, the model computes a supply-demand (partial) equilibrium, where both the supply side options and energy service demands are endogenous. Endogenisation of energy demands is via user-defined demand functions, which allow them to be responsive to their own price. Based on economic theory (Equivalence Theorem), the equilibrium reached equates to the maximization of the net total surplus, ${ }^{8}$ defined as the sum of producer and consumers surplus, net of production costs (Loulou et al. 2005).

Maximising net total surplus means that the model is no longer solely minimising system costs (as in the standard version of ESME) but rather capturing a broader metric, which includes a measure of societal welfare. In effect, it is going beyond the energy system boundary by capturing insights on consumers' willingness to pay for energy services versus other goods and services. The maximisation problem can be written as follows (Loulou et al. 2005) -

$$
\begin{aligned}
\operatorname{Max} \sum_{i} & \sum_{t}\left(p_{i}^{0}(t) \cdot\left[D M_{i}^{0}(t)\right]^{-1 / E} \cdot D M_{i}(t)^{1+1 / E} /\left(1+1 / E_{i}\right)\right)-c \cdot X \\
\text { s.t. } & \sum_{k} C A P_{k, i}(t) \geq D M_{i}(t) \quad i=1, \ldots, I ; t=1, \ldots, T \\
& B \cdot X \geq b
\end{aligned}
$$

Where equation 1 is an expression of the total surplus to be maximised. It expresses that system costs should be minimised, that is all supply side variables $(X)$ and their associated costs $(c)$ whilst simultaneously allowing for consumer surplus losses based on the cost $(p)$ of demand side reductions (DM). Equation 2 specifies the requirement to meet energy service demands while equation 3 represents all other constraints in the problem. Demand functions provide the basic mechanism for modelling the price elasticity of energy service demands, and can be expressed as follows:

$$
D M_{i, t, x}^{0} / D M_{i, t, x}=\left(p_{i, t, x}^{0} / p_{i, t, x}\right)^{E_{i, t, x}}
$$

Where $D M$ is the energy service demand, $p$ is the price of energy service demand, and $E$ is the (negative) elasticity. (DM ${ }^{0}, \mathrm{p}^{0}$ are the reference demand and price for a given energy service demand). Crucially,

\footnotetext{
8 In this case, net total surplus represents the aggregate of producer surplus (gross profit, based on total revenue minus production costs) and consumer surplus (the opportunity gain of purchasing at a level lower than price one is willing to pay).
} 
these demand functions can be approximated by using piece-wise linear functions, ensuring the mathematical programme remains linear, reducing complexity and ensuring comparable solution times. Demand curves are integrated into the model for each combination of end-use demand service $(x)$, model year $(t)$, and node $(i)$.

The first input to constructing the demand curve is the marginal price of an energy service demand (for a given node and period), calculated by running the model in its non-elastic formulation. This is the reference price around which the demand curve is built. Three further parameters are required to define the demand curve; first, the elasticity factor determining the responsiveness of demand to price, second, the number of steps to linearise the demand curve ${ }^{9}$ and third, the limit on the percentage demand change.

\subsubsection{Integrating elasticity values into the ESME model}

The strength of this analysis is using a probabilistic approach to capture the uncertainties across price elasticities. The demand elasticity ranges were implemented into the model as triangular probability distributions. The use of this probability distribution type reflects the limited spread of values from the literature review, allowing for use of a mode, min and max value, and an asymmetric distribution. Other distributions were considered but deemed unsuitable due to the need for more data points, or simply due to practical problems of non-compatibility in the ESME model framework. Elasticity values, for all demands, were considered to be symmetrical (increase / decrease at same rate for a given price change) although in practice, under a low carbon analysis, prices tend to increase resulting in response in one direction, a demand reduction. Elasticity values were also held constant over time, assuming that consumer response to price is not affected in future years by other factors, such as the impact of price increases across other non-energy goods, changes in income etc.

\footnotetext{
${ }^{9}$ The greater the number of steps used to linearise the demand curve, the closer the approximation to the non-linear form, and the greater the flexibility of the model in terms of incremental movement up and down the curve. However, model testing shows that after a certain point, increasing the number of steps does not discernibly impact on demand response choice.
} 
Prior to sampling probability distributions in AIMMS using a Monte Carlo approach, some of the energy services were correlated, to reflect that specific demands broadly provide the same energy services to the same population groups, and therefore elasticity value should not diverge significantly. This was the case for car demands (perfect positive correlation) and dwellings (strong positive correlation), where we would expect the behaviour of similar respondents (car owners, households) to be strongly correlated under a given simulation. Rail and bus demands were negatively correlated (to a moderate extent), to indicate that a high demand reduction is less likely across both demands under a given simulation.

\section{Results}

\subsection{Model set-up and runs}

The analysis undertaken aims to analyse demand response under carbon constraints $\left(\mathrm{CO}_{2}\right.$ emissions only). Three scenarios were set up - Reference, High Growth and Low Growth cases - all constrained to meet the UK's $80 \%$ carbon reduction targets (relative to 1990 levels) plus interim targets (or carbon budgets) between 2020 and 2050 (DECC 2011). High and low growth cases were chosen to assess the role of demand response under different levels of target stringency (for example, a given target is much more stringent under a high demand case). Reference case energy service demands reflect UK government central projections for GDP, population and households, complimented by specific transport projections from the Department for Transport (DfT 2010) and industry projections from the Department for Energy and Climate Change (DECC). The Low and High Growth cases assume GDP growth rates of $2.1 \%$ and $3.4 \%$ respectively, compared to the Reference growth rate of $2.7 \%$.

For each scenario, the model was run deterministically without a carbon constraint, generating energy demand marginals to calibrate demand curves used in the elastic demand formulation. Correlated samples were generated from probability distributions for price elasticities using a Gaussian copula. Sampled values were then used in 500 simulation runs; the number of simulations adequately covering the uncertainty space were estimated based on the approach in Morgan and Small (1992) for a 95\% confidence interval. For all three scenarios, uncertainty associated with other supply side input 
parameters was switched off (held deterministic) in order to closely scrutinise the demand response uncertainty.

\subsection{Model results: Reference scenario}

Reference scenario results highlight that demand reductions under carbon constraints can greatly reduce abatement costs compared to a case where no demand response is modelled, as shown in Figure 1. Loss of welfare associated with demand reductions is estimated to be a lower cost mitigation intervention than many supply side options, reflecting an important trade-off. There is also an important temporal dimension observed, with higher demand response in 2030 and 2040, indicating that this is an important transition mechanism on the pathway to a lower carbon system, as stringency of emission targets increases and supply side technologies are not yet cost-effective. Under this perfect foresight modelling framework, the optimal deployment of demand reduction can be achieved. In 2020, while abatement costs increase, this actually reflects increasing use of demand response to avoid early deployment of low carbon technologies.

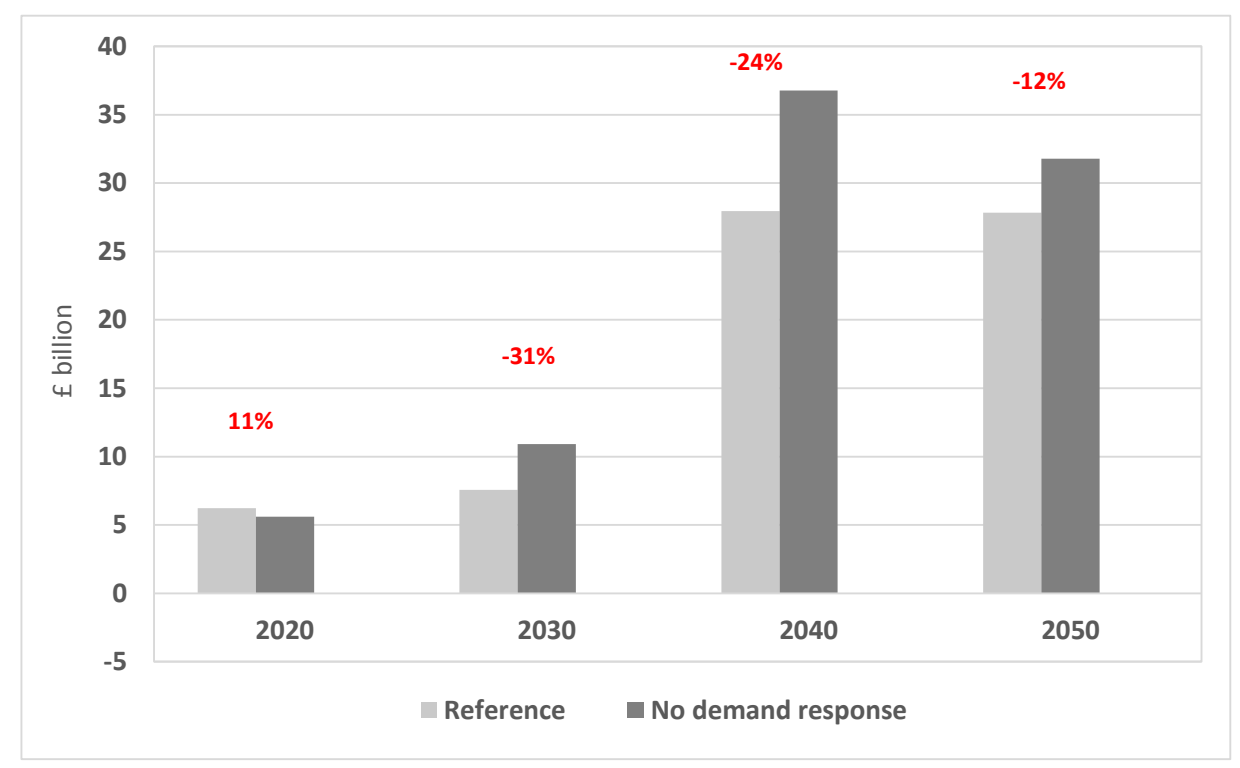

Figure 1. Abatement costs for Reference case (average of 500 simulations) compared to a no demand response case. Red values denote change in abatement cost attributed to demand response. 
While Figure 1 provides the average picture, what about the impact of price response uncertainty? Broadly speaking, demand response always plays a key role in reducing mitigation costs. For example, in 2040 demand reduction drives down abatement costs by between 22 and $26 \%$, in $90 \%$ of cases (Figure 2). In 2030, the spread of reduction in abatement costs is greater but nevertheless implies a strong role for demand response in all cases. The key insight for policy is that measures that can affect behaviour have an important role to play in reducing mitigation costs, particularly during the period of transition to a low carbon system. This is also reflected by the marginal costs of abatement, which drop to $£ 251$ and $£ 396 / \mathrm{tCO}_{2}$ in 2030 and 2040, compared to $£ 346$ and $£ 479 / \mathrm{tCO}_{2}$ under the no demand response case. These reductions are of course predicated on the systems valuation of welfare losses associated with demand reduction - and how these compare to supply side costs, which in themselves can be highly uncertain (as discussed later).

Driving the demand response under climate targets is the transport sector, reflecting both the greater sensitivity to price changes in the sector and the higher supply side costs of mitigation. Another important factor is also at play; the buildings sector has a much lower demand response as a result of the increasing use of low carbon electricity; on average, electricity use in buildings grows from $17 \%$ in 2020 to $53 \%$ in 2050 . Higher carbon prices do not therefore drive demand response in this sector as there is limited scope for further decarbonisation by doing so. In 2050, the transport sector accounts for $83 \%$ of consumer surplus losses, the valuation of demand reduction in the model, compared to $15 \%$ in buildings. The industry sector accounts for the remaining share of demand response, at a very low level due to the assumption of demand being price inelastic. These shares are also observed across earlier time periods. 

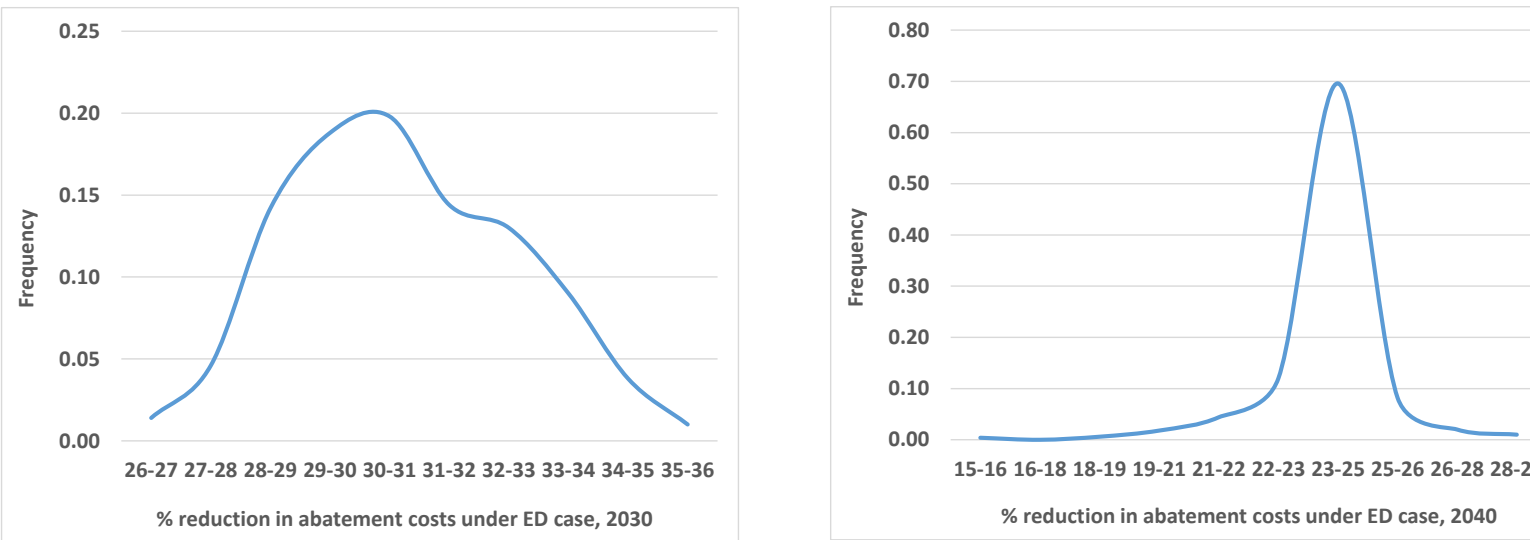

Figure 2. Distribution of reductions in abatement costs for Reference case in 2030 and 2040 (for 500 simulations) compared to case with no demand response.

A standard deviation of $2.5 \%$ for transport sector contribution to consumer surplus losses across all periods suggest that uncertainty associated with price elasticity does not strongly impact on sectoral contribution. Specifically, it is car demand where the highest share of consumer surplus loss is observed, although the uncertainties are more evident, with a 25 to 75 percentile range of $32-40 \%$, and standard deviation of $5.5 \%$ (see Figure 3). For those simulations where car demand response is low (due to more inelastic price elasticities), other transport demands see increased demand reductions, broadly maintaining overall sectoral contribution. While car demand has the single biggest impact of any single demand, the cumulative contribution of all other transport demands is higher in the majority of simulations.

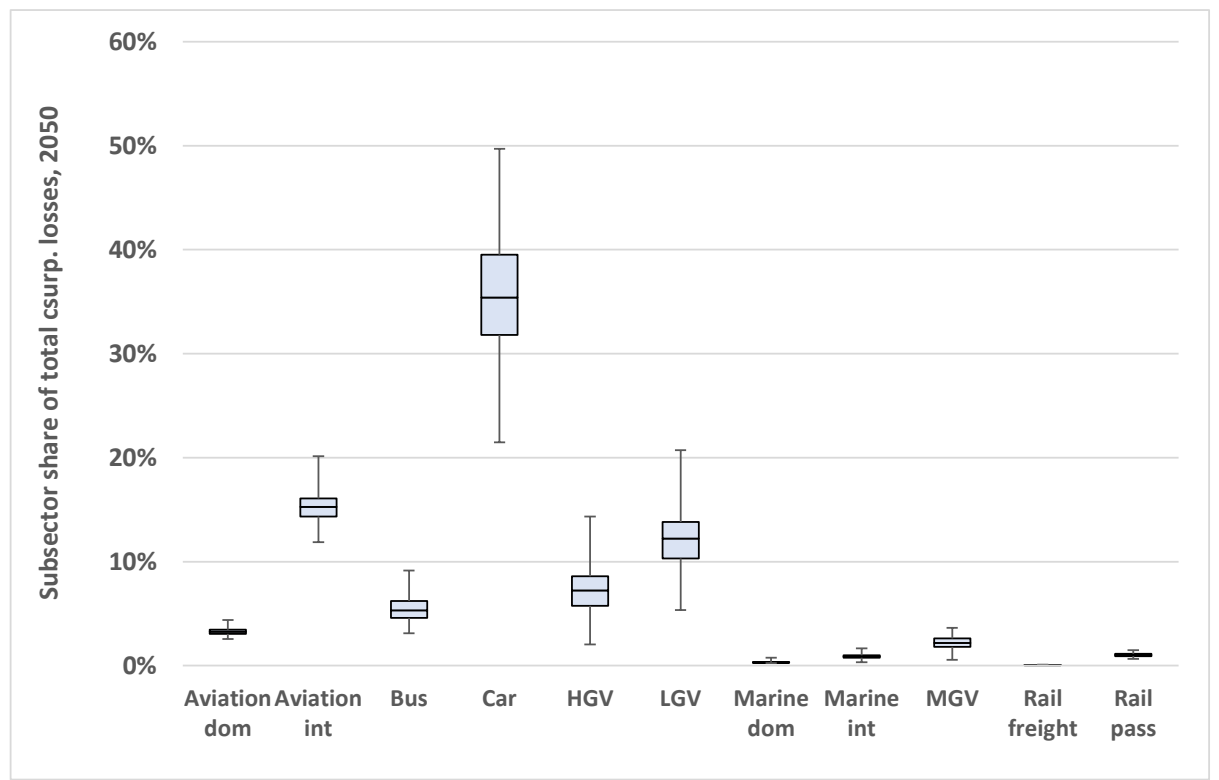

Figure 3. Box-and whisker plot of the share of total system wide consumer surplus losses by transport subsector in 2050 
The analysis strongly indicates that behavioural measures should be focused in the transport sector. While private car travel reduction features strongly in many simulation, other transport modes also feature where car travel demand is assumed more inelastic to price increases. For policymakers, this is challenging as it points to formulating behavioural policies in a sector considered quite intransigent to change. Actual reductions in energy service demands (in physical units) are shown in Figure 4. Car demand, an important driver of consumer surplus losses, has a relatively small reduction in demand compared to other demands. This highlights two features of car demand; it is the largest single transport demand so small reductions still have a big impact on the system, and secondly, the unit cost of car demand is higher than for other transport demands. This latter point is important, as it illustrates how important characterisation of the cost of energy service demand is in this type of analysis (for the calibration and valuation of demand response), including consistency in cost characterisation across demands.

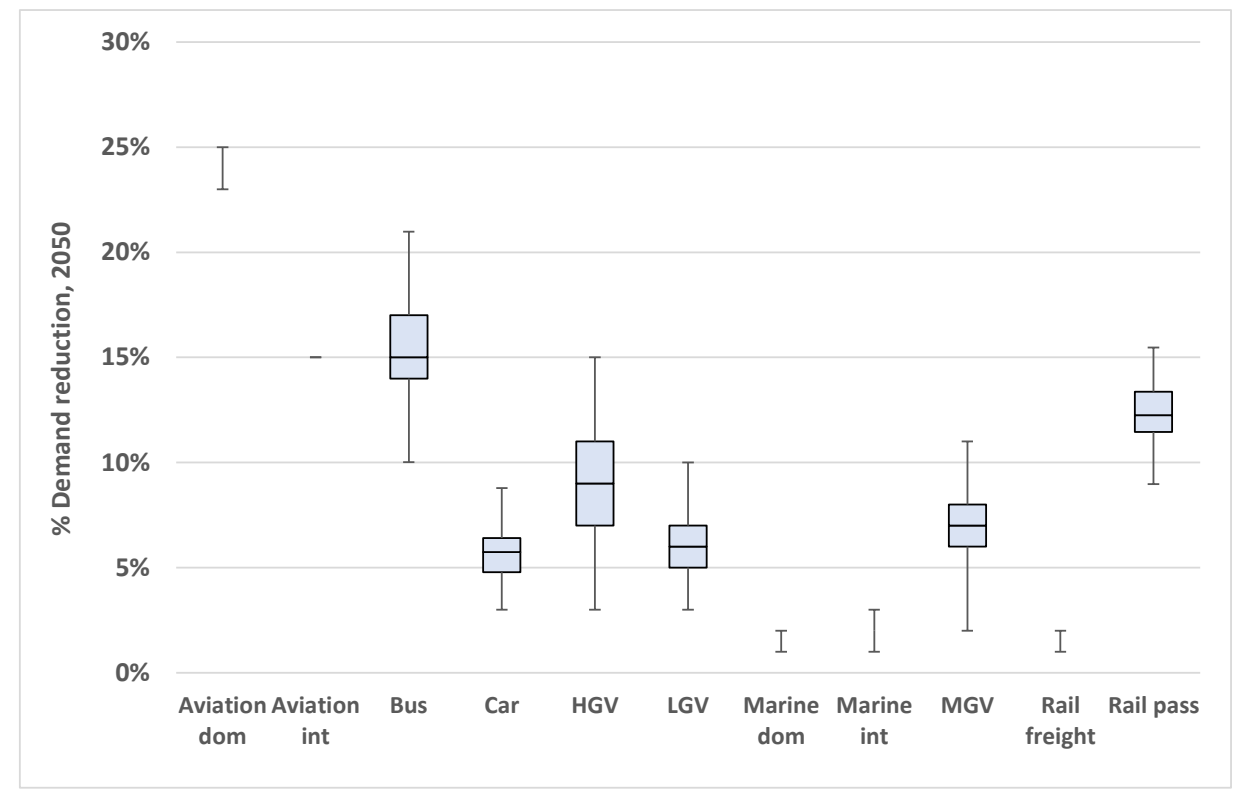

Figure 4. Box-and whisker plot of \% demand reduction by transport subsector in 2050

The demand reductions provide headroom for emissions growth, resulting in a shift in supply side mitigation (Figure 5). In 2030, reduction in demands reduce emissions from the transport sector, allowing for greater use of gas for heating in buildings. A reduction in the use of hydrogen in the transport sector shifts CCS from hydrogen production to industry. Overall, there is no net change in 
total emissions but a 15\% swing in mitigation source type contribution. This swing illustrates the impact of demand response on supply side mitigation choices, in this case changing mitigation choice across $15 \%$ of total emissions. A similar pattern is observed in 2050. Across the simulations, the pattern of supply side changes is relatively stable, reflecting the deterministic modelling of supply side assumptions. Under lower demand responses, the percentage swing reduces; however, the change in mitigation choices remains the same. These results provide an interesting insight into where technology options lie on the system wide cost curve, and the role demand reduction can play in shifting supplyside mitigation down the curve, reducing marginal abatement costs.

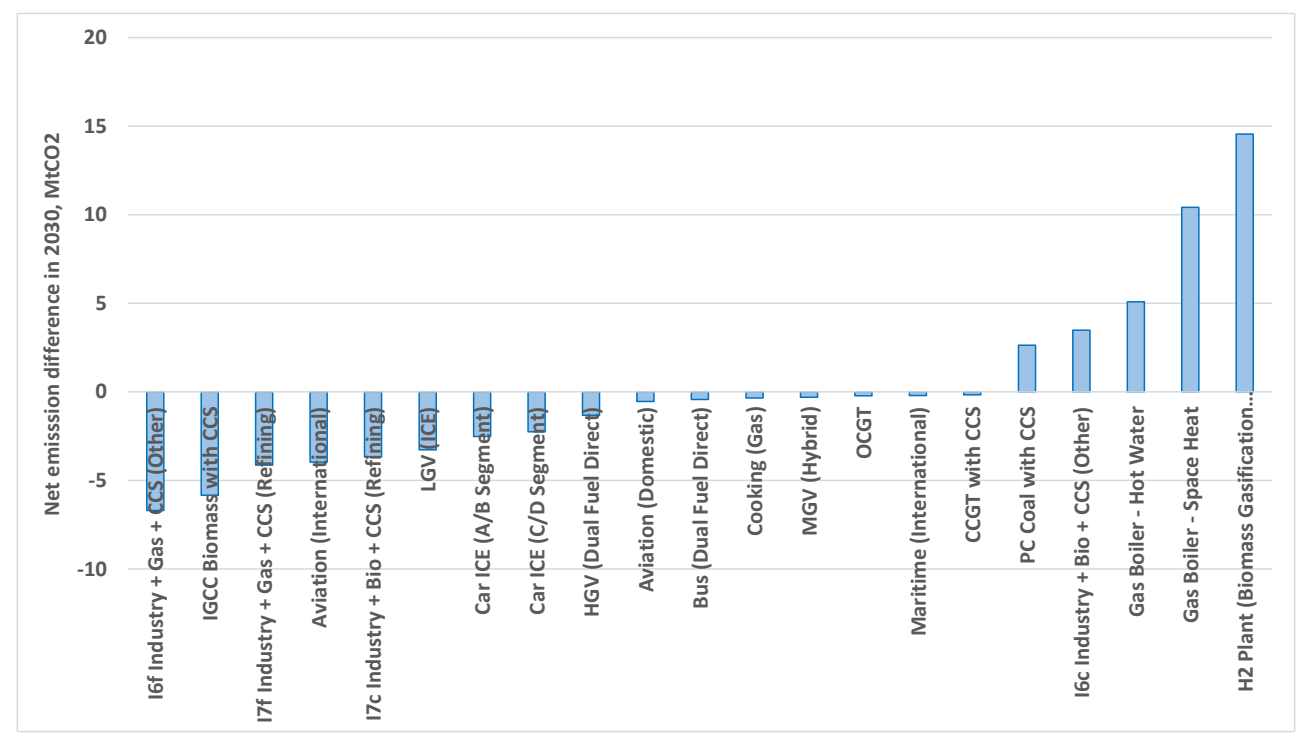

Figure 5. Difference in selected emission sources between Reference cases (on average) and equivalent without demand response case in 2030

The analysis highlights that demand reduction allows for a more gradual, cost-effective transition to a low carbon system. It means that the uptake rate of different low carbon technologies can be slowed, 'buying' more time for technology development and infrastructure build. However, this should not be seen as a signal that complacency can be afforded in the transition but rather that strong behaviouralbased policies, if put in place, allow for a more cost-effective transition.

\subsection{Model results: Comparison with High and Low growth scenarios}

The High Growth scenario reflects increasing stringency of future emission reduction targets, resulting in a larger demand response in absolute terms (compared to the Reference case). This is particularly true in the earlier model periods ( $-43 \%$ in $2020,-30 \%$ in 2040$)$, given the higher cost of supply side 
technologies, and the weighting of near term costs under the social discount rate. The spread of abatement reduction values is marginally wider than observed in the Reference case; however, the standard deviation from the mean is less than $2 \%$, highlighting the key role for demand response in most simulations. In the low growth case, demand response plays an equally important role, despite the relative stringency of carbon reduction targets being less. Abatement costs reduce by over $30 \%$ in 2020 and 2040, but are significantly higher in $2030(+39 \%)$.

The transport sector remains the key driver of consumer surplus losses under both the low and high growth case. In the high growth case, however, there is more uncertainty (albeit a small increase) concerning the sector's contribution, with the building sector contribution on average higher with a larger range. Within the transport sector, the average level and range of road and rail transport demand responses increase compared to the Reference case (Figure 6). Higher carbon prices both drive larger demand reductions and give rise to greater uncertainty in transport subsector contribution.

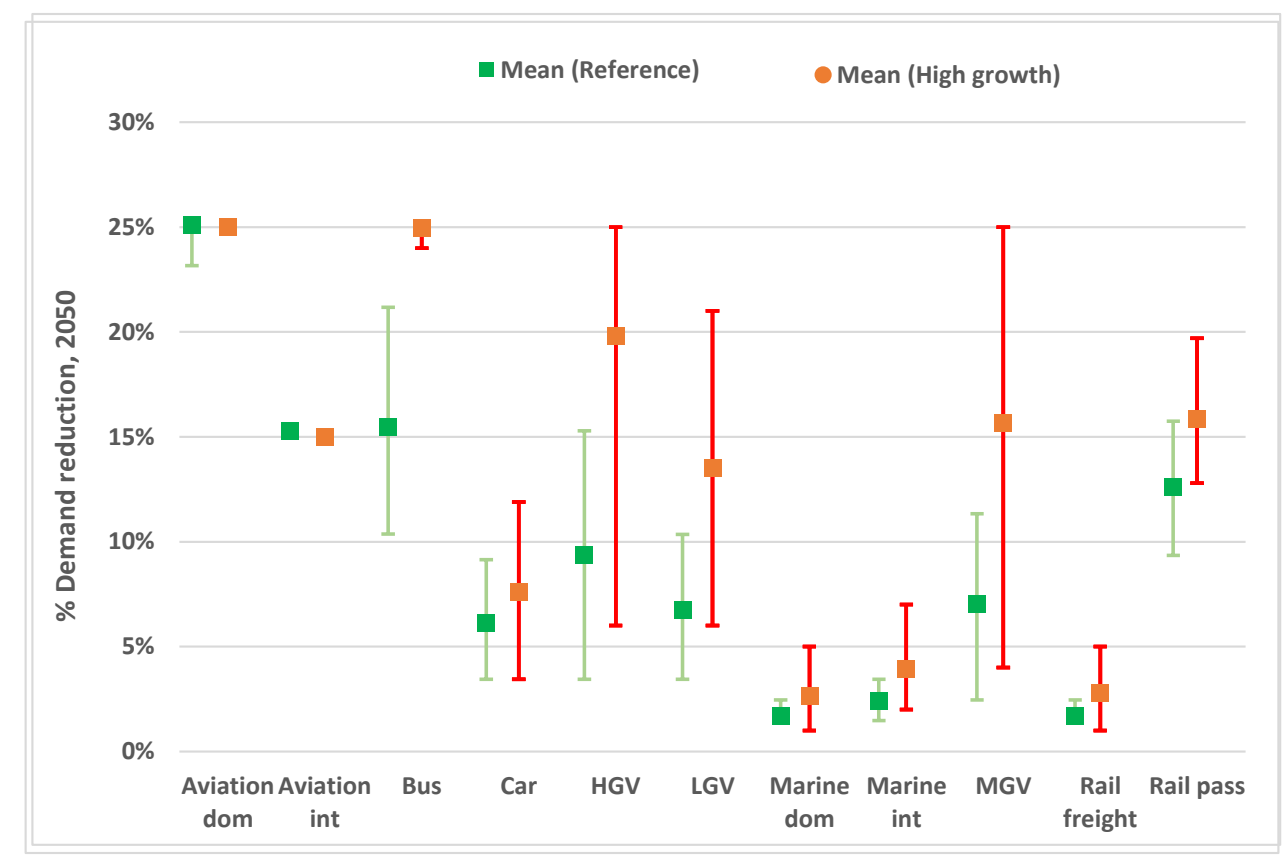

Figure 6. \% demand reduction in 2050 by transport subsector across Reference and High Growth cases (Error bars indicate range)

Road freight in particular shows a larger demand reduction on average, and a larger range of possible outcomes. The impact on consumer surplus losses is now not so predominantly driven by car demand reduction in later periods, with LGV / HGV demand reduction playing as strong a role in terms of abatement costs. On average (compared to the Reference case), \% contribution to overall consumer 
surplus losses reduces for cars (from 35\% to 25\%) and increases for HGVs and LGVs (7\% to $15 \%$, and $12 \%$ to $17 \%$ respectively). While the wider spread of demand responses observed in Figure 6 reflect greater uncertainty with respect to where demand reductions may occur within the sector, the insight that it is the transport sector where most demand reduction will occur still stands, accounting for on average between $70-80 \%$ of consumer surplus losses observed across the time periods.

The High Growth case shows that supply side trade-offs are much more pronounced for the power sector in 2050 and the transport sector in 2030; the reverse of this trend is observed in the Reference case. This results, from an even stronger focus on reducing costs in earlier periods, and therefore targeting transport in 2030. The difference in technology choices across simulations is limited, with relatively low deviation from the mean. Due to the higher demand response in the buildings sector under the High Growth case, a stronger impact on the electricity generation sector is observed. In 2050, an average $17 \%$ net reduction in generation in the High Growth case compares to $9 \%$ in the Reference case, and highlights that a range of low carbon technologies are not used as a result of a reduction in demand, particularly offshore technologies (Figure 7). Tidal and wave technologies appear in no simulations whilst offshore wind appears in less than $1 \%$ of simulations.

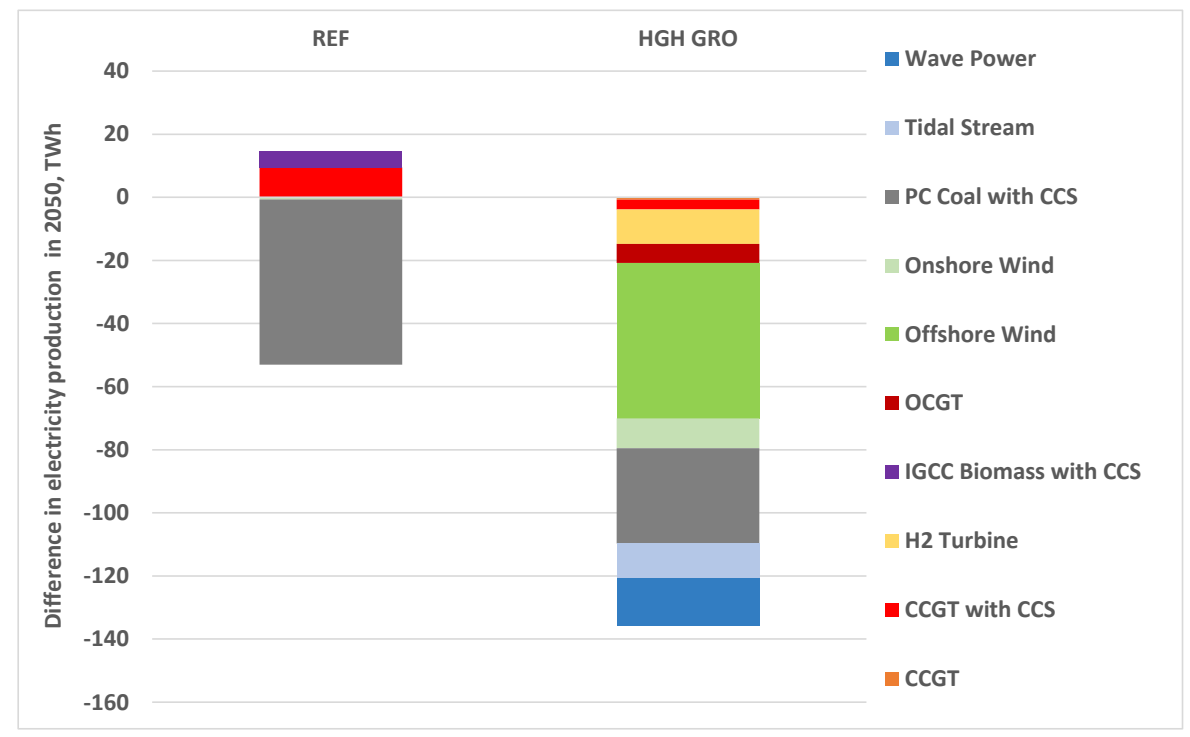

Figure 7. Difference in electricity production by type between high growth and Reference $\mathrm{CO}_{2}$ reduction cases (average) and equivalent without demand response cases in 2050 


\section{Discussion}

Price-driven reductions in demand play an important role in ensuring a more cost-effective transition to a low carbon economy. Uncertainty concerning price responsiveness in different sectors can have some impact on the reductions in mitigation costs; however, this uncertainty does not weaken the key insight that demand response is always an important measure for emission mitigation. While demand reductions are observed across the transition pathway, they have a particularly strong role to play in the near to medium term, when the cost of low carbon technologies are still relatively high and their deployment is limited. There is therefore a persuasive case that government strategy should be more focused on demand side measures in the near to medium term, to allow for cost-effective rates of low carbon technology uptake.

Another important policy insight is that the focus of demand response, from a system wide context, is the transport sector. The probabilistic approach allows us to conclude that this finding is robust, and is due to a number of reasons; firstly, the review of the literature suggests that transport demands are more elastic (in general), secondly, the costs of supply-side mitigation in the transport sector are higher and thirdly, with decarbonisation of the power sector a usual route for a cost-effective pathway, higher use of low carbon electricity in non-transport sectors reduces the carbon intensity of energy demand, in turn leading to a more limited role for demand response. Higher levels of demand response are observed in the transport sector where average carbon intensity of energy is higher. In a system where decarbonised electricity has high rates of penetration into the vehicle fleet, demand response may be more limited. However, preliminary testing of different low carbon system configurations does not necessarily support this premise. Further analysis is required here although one reason may be the high costs of additional transport system electrification (additional low carbon generation, infrastructure, vehicle costs) versus the welfare loss associated with demand reductions.

The challenge for policy is how to achieve the observed reductions in the transport sector while not curtailing travel choices for the population. In addition, what are the costs of the policies needed to deliver these reductions, which are excluded from this analysis, and would this undermine costeffectiveness of such actions? Strong price focused policies will certainly be needed to deliver the 
reduction levels observed, and are likely to be politically sensitive. With demands, such as aviation, hitting the reduction limit imposed in the model, there remains a question concerning what could be achieved in the real system, given the intransigence of habit, and limited alternatives depending on the geography and purpose of travel. ${ }^{10}$ While the analysis points to a strong tendency toward demand reductions in the transport sector, the specific subsector in which this occurs is less certain; as car travel demand price elasticities become increasingly inelastic, demand reduction in other transport sectors increases. This ensures that the aggregate transport sector demand response is always more than $80 \%$ of total consumer surplus losses. These uncertainties mean that understanding which transport subsectors are best to target with policy measures is challenging.

The analysis also highlights some interesting trade-offs between demand side measures and supply side investments. In effect, lower demand creates emission headroom (relative to a case with no demand response), allowing for a more gradual uptake of low carbon technologies, and resulting in a more costeffective transition. This should not suggest that supply side focused policy need be less ambitious but rather that cost-effective opportunities can be realised by incentivising consumers to reduce demand, through price incentives / mechanisms as well as supply-side conservation and efficiency measures.

Under more stringent targets, an even stronger role for demand reductions is observed. Key insights from the High Growth scenario are two fold; higher economic growth is going to make decarbonisation more costly, highlighting an even stronger role for cost-effective demand reduction measures to increase affordability. Secondly, technologies that are removed from the solution should just be indicative of the role in demand reduction reducing marginal abatement costs, not specific technology winners and losers (as discussed earlier). While not providing significant new insights, the low growth case does underline the strong role of demand reduction even under a less stringent carbon reduction case.

\section{Conclusions and Policy Implications}

The objective of this paper was two-fold; first to use more robust elasticity estimates via a literature review. The second was to consider the uncertainty associated with this important input parameter on

\footnotetext{
${ }^{10}$ Goodwin (2007) notes that the evidence available is rich concerning reductions in car use up to about $20 \%$ or $30 \%$, but very sparse beyond that.
} 
the model outputs. The review in this paper provides a stronger empirical basis for the use of price elasticities in ESME and other energy systems models. The international nature of the literature reviewed means these estimates can provide a useful basis for models in comparable OECD countries. However, from the review it is also evident that further primary research is needed to understand demand response across sectors, notably buildings, industry and transport freight.

Estimates used in previous UK ESM analyses (with a poorly understood empirical basis) have tended to suggest the transport sector has a low demand response, with other sectors between $15-20 \%$, including industry (AEA 2008, Anandarajah et al. 2009, AEA 2011). This analysis reveals some new and quite different insights - a stronger role for the transport sector, and for mitigation action in the near to mid-term (during the transition), as a cost-effective option which provides temporal flexibility in the deployment of new low carbon technologies. The review also highlights the difficulty in characterising industrial demand response in this type of modelling framework that only account for energy costs and does not consider competitiveness impacts.

With regard to the second objective, the uncertainty across estimates used in this analysis does not undermine the critical aggregate role of this mechanism in climate mitigation, despite being wide ranging for some energy service demands. Uncertainties do give rise to differences in abatement cost levels, the role of different transport sectors, and supply side trade-offs are observed; however, the strong role for demand reductions is evident under most simulations, providing a robust finding for policy makers. Ongoing analysis suggests that introducing wider system uncertainties (on the supply side) has a limited impact on the role of demand response, reflecting that demand curves are calibrated to supply side costs (for each simulation), and are therefore always built around energy service marginals, calculated based on the relative costs of supply side options. Further analysis is required to understand what other uncertainties in the system, not currently captured in ESME, could result in lower demand response, and the extent to which system configuration (and transition pathways) entrench the critical importance of demand reductions.

A number of themes for continuing research emerge from this paper. Firstly, further consideration should be given to how price elasticities are incorporated into ESMs. The literature review identifies a 
wide range of price elasticities depending on the type of demand (e.g. business versus leisure), location of demand (rural versus urban), timing of demand (off peak versus peak) and demand groups (low versus high income). These distinct responses are at best averaged in this analysis, at worst leading to missed insights. Further research should re-consider the balance between demand disaggregation and generating new model insights. Secondly, further consideration should be given to modelling modal shift, to provide additional insights concerning whether transport demand for a given mode reduces or shifts to a different mode. This could be done via cross-price elasticities or using other mechanisms, such as through using time budget constraints and mode speed, capturing other aspect of travel utility (Schafer 2012).

Thirdly, further research is needed across different sectors (primarily non-transport) on demand responsiveness to price and the absolute levels of reduction that could be feasibly achieved. In part, this could be down to the role of telecommunication as an alternative to travel (Mokhtarian 2002). The role of supply side model configuration e.g. high electrification pathway, should also be further analysed, to see what impact this might have on the uptake of demand reduction. Fourthly, the sensitivity of models to uncertainty should be tested, via sensitivity analysis, to explore where demand response uncertainties rank against other model uncertainties. This could be done via global sensitivity analysis, defined as the study of how uncertainty in the output of a model can be apportioned to different sources of uncertainty in the model input (Saltelli et al. 2008). 


\section{References}

AEA (2011). Pathways to 2050 - Key Results. A Report to the Department of Energy and Climate Change. May 2011. AEA, London.

https://www.gov.uk/government/uploads/system/uploads/attachment data/file/48072/2290-pathwaysto-2050-key-results.pdf

AEA (2008). MARKAL-MED model runs of long term carbon reduction targets in the UK, Phase 1. Authored by Pye, S., N. Hill, T. Palmer, and N. Ozkan. On behalf of the Committee on Climate Change. November 2008. http://www.theccc.org.uk

Anandarajah, G., Strachan, N., Ekins, P., Kannan, R., and Hughes, N. (2009). Pathways to a low carbon economy: energy systems modelling. UK Energy Research Centre. http://www.ukerc.ac.uk/

Bhattacharyya, S., \& Timilsina, G. R. (2009). Energy demand models for policy formulation: a comparative study of energy demand models. World Bank Policy Research Working Paper Series, Research Paper 4866.

Bernstein, M. A., and Griffin, J. M. (2006). Regional differences in the price-elasticity of demand for energy. National Renewable Energy Laboratory.

Boonekamp, P. G. (2007). Price elasticities, policy measures and actual developments in household energy consumption-A bottom up analysis for the Netherlands. Energy Economics, 29(2), 133-157.

CCC (2013). Reducing the UK's carbon footprint and managing competitiveness risks. Committee on Climate Change, April 2013. http://www.theccc.org.uk/

CCC (2013). Fourth Carbon Budget Review - part 2: The cost-effective path to the 2050 target.

Committee on Climate Change. December 2013. http://www.theccc.org.uk/

CCC (2011). The Renewable Energy Review. Committee on Climate Change, May 2011. http://www.theccc.org.uk/

Chen, W., Wu, Z., He, J., Gao, P., \& Xu, S. (2007). Carbon emission control strategies for China: a comparative study with partial and general equilibrium versions of the China MARKAL model. Energy, 32(1), 59-72.

Dargay, J. (2010). The prospects for longer distance domestic coach, rail, air and car travel in Britain. Report by Institute of Transport Studies, Leeds, to the Independent Transport Commission, January 2010.

Dargay, J. (2007). The effect of prices and income on car travel in the UK. Transportation Research Part A: Policy and Practice, 41(10), 949-960.

Dargay, J., Menaz B., and Cairns S. (2006). Public Attitudes towards Aviation and Climate Change, Stage I: Desktop Research. Report prepared for Commission for Integrated Transport, October 2006.

Dargay, J. M., \& Hanly, M. (2002). The demand for local bus services in England. Journal of Transport Economics and Policy, 73-91.

DECC (2011). The Carbon Plan: Delivering our low carbon future. Department of Energy and Climate Change. December 2011.

https://www.gov.uk/government/uploads/system/uploads/attachment_data/file/47613/3702-thecarbon-plan-delivering-our-low-carbon-future.pdf

De Jong, G., \& Gunn, H. (2001). Recent evidence on car cost and time elasticities of travel demand in Europe. Journal of Transport Economics and Policy, 137-160. 
DfT (2013). UK Aviation Forecasts. Department for Transport. January 2013

https://www.gov.uk/government/uploads/system/uploads/attachment_data/file/223839/aviation-

forecasts.pdf

DfT (2010). Road Transport Forecasts 2009 - Results from the Department for Transport's National Transport Model, Department for Transport. March 2010 http://webarchive.nationalarchives.gov.uk/20110202223628/http://www.dft.gov.uk/pgr/economics/nt m/forecasts2009/pdf/forecasts2009.pdf

Ekins, P., Keppo I., Skea J., Strachan N., Usher W., Anandarajah G. (2013). The UK Energy System in 2050: Comparing Low-Carbon, Resilient Scenarios. (UKERC Report UKERC/RR/ESY/2013/001). UK Energy Research Centre: London, UK. http://www.ukerc.ac.uk/

Fishbone, L. G., \& Abilock, H. (1981). Markal, a linear-programming model for energy systems analysis: Technical description of the bnl version. International journal of Energy research, 5(4), 353375.

Goodwin, P. (2007). Carbon abatement in transport: Review of demand responses. Undertaken on behalf of the Committee on Climate Change (CCC), December 2007

Goodwin, P., Dargay, J., \& Hanly, M. (2004). Elasticities of road traffic and fuel consumption with respect to price and income: a review. Transport Reviews, 24(3), 275-292.

Graham, D. J., \& Glaister, S. (2004). Road traffic demand elasticity estimates: a review. Transport reviews, 24(3), 261-274.

Guertin, C., Kumbhakar, S. C., \& Duraiappah, A. K. (2003). Determining demand for energy services: investigating income-driven behaviours. International Institute for Sustainable Development.

Haas, R., Auer, H., \& Biermayr, P. (1998). The impact of consumer behavior on residential energy demand for space heating. Energy and buildings, 27(2), 195-205.

Hourcade, J. C., Jaccard, M., Bataille, C., \& Ghersi, F. (2006). Hybrid modeling: New answers to old challenges. The Energy Journal, 2(Special issue), 1-12.

IEA (2012). Energy Technology Perspectives 2012 - Pathways to a Clean Energy System. International Energy Agency, Paris. ISBN: 978-92-64-17488-7.

InterVISTAS (2007). Estimating Air Travel Demand Elasticities. Prepared for IATA. December 2007. http://www.iata.org/whatwedo/Documents/economics/Intervistas Elasticity Study 2007.pdf

Kannan, R., Strachan, N., Pye, S., Balta-Ozkan, N., (2007). UK MARKAL Model Documentation. www.ukerc.ac.uk

Kesicki, F., \& Anandarajah, G. (2011). The role of energy-service demand reduction in global climate change mitigation: Combining energy modelling and decomposition analysis. Energy Policy, 39(11), 7224-7233.

Klein, Y. L. (1988). An econometric model of the joint production and consumption of residential space heat. Southern Economic Journal, 351-359.

Litman, T (2013). Understanding Transport Demands and Elasticities: How Prices and Other Factors Affect Travel Behavior. Victoria Transport Policy Institute. March 2013.

Loulou, R., Remme U., Kanudia A., Lehtila A., Goldstein G. (2005). Documentation for the TIMES Model. Energy Technology Systems Analysis Programme (ETSAP). April 2005. 
Loulou, R., \& Lavigne, D. (1996). MARKAL model with elastic demands: application to greenhouse gas emission control (pp. 201-220). Springer Netherlands.

Messner, S., \& Schrattenholzer, L. (2000). MESSAGE-MACRO: linking an energy supply model with a macroeconomic module and solving it iteratively. Energy, 25(3), 267-282.

Mokhtarian, P. L. (2002). Telecommunications and travel: The case for complementarity. Journal of Industrial Ecology, 6(2), 43-57.

Molloy, J., Melo, P. C., Graham, D. J., Majumdar, A., \& Ochieng, W. Y. (2012). Role of Air Travel Demand Elasticities in Reducing Aviation's Carbon Dioxide Emissions. Transportation Research Record: Journal of the Transportation Research Board, 2300(1), 31-41.

Morgan, M. G., \& Small, M. (1992). Uncertainty: a guide to dealing with uncertainty in quantitative risk and policy analysis. Cambridge University Press.

Nakićenović, N. (Ed.). (2012). Global Energy Assessment: Toward a Sustainable Future. Cambridge University Press.

Paul, A., Myers, E., \& Palmer, K. (2009). A partial adjustment model of US electricity demand by region, season, and sector. Resource for the Future. DP-08-50.

Paulley, N., Balcombe, R., Mackett, R., Titheridge, H., Preston, J., Wardman, M. \& White, P. (2006). The demand for public transport: The effects of fares, quality of service, income and car ownership. Transport Policy, 13(4), 295-306.

RCEP (2000). Energy - The Changing Climate. 22nd report of the Royal Commission on Environmental Pollution. London. TSO.

Saltelli, A., Ratto, M., Andres, T., Campolongo, F., Cariboni, J., Gatelli, D., \& Tarantola, S. (2008). Global sensitivity analysis: the primer. Wiley.com.

Schafer, A. (2012). Introducing behavioral change in transportation into energy / economy / environment models. World Bank Policy Research Working Paper, (6234).

Seebregts, A. J., Goldstein, G. A., \& Smekens, K. (2002). Energy/environmental modeling with the MARKAL family of models. InOperations Research Proceedings 2001 (pp. 75-82). Springer Berlin Heidelberg.

Sorrell, S., Dimitropoulos, J., \& Sommerville, M. (2009). Empirical estimates of the direct rebound effect: A review. Energy policy, 37(4), 1356-1371.

Sorrell, S., and Dimitropoulos J. (2007). UKERC Review of Evidence for the Rebound Effect, Technical Report 2: Econometric Studies. UK Energy Research Centre: London.

http://www.ukerc.ac.uk/support/ReboundEffect

Sorrell, S. (2007). The Rebound Effect: an assessment of the evidence for economy-wide energy savings from improved energy efficiency. London: UK Energy Research Centre.

http://www.ukerc.ac.uk/support/ReboundEffect

Strachan, N., Pye, S., \& Kannan, R. (2009). The iterative contribution and relevance of modelling to UK energy policy. Energy Policy, 37(3), 850-860.

Strachan, N., Foxon, T., \& Fujino, J. (2008). Policy implications from the Low-Carbon Society (LCS) modelling project. Climate Policy, 8(sup1), S17-S29. 